\section{Molecular Detection of Mycobacterium tuberculosis (MTB) and Rifampicin Resistant Strain among Subjects Accessing Health Care at Federal Medical Centre, Yenegoa, Bayelsa State; Nigeria}

\section{Abstract}

Background: An increasing global incidence of Mycobacterium Tuberculosis (MTB), and emergence of drug resistant strains of MTB, have critically promoted the urgent need to explore better effective methods that can rapidly detect MTB, and identify drug-resistant cases as recommended by World Health Organization report of 2008. Globally, multi-drug-resistant strains of MTB are increasingly becoming a huge public health issue, which has adversely affected the health care delivery of patients, especially in developing communities with limited functioning health care facility. Nonetheless, the aim and focus of the study is to evaluate the trend of rifampicin resistance strains of MTB among patients accessing care at the Federal Medical Centre Yenegoa, Bayalsa State.

Methods: This observational cross-sectional study which utilized stratified proportionate random technique, was designed to evaluate the molecular detection, as well as the prevalence of MTB and multidrug resistant TB specifically RIF among the studied population.

Results: The study population were 204 subjects recruited randomly, with a mean age of $37.23 \pm 16.68$ years and were categorized based on sex, $93(45.6 \%)$ males and 111 (54.4\%) females respectively. The MTB detected were 21 (10.3\%) while 183 (89.7\%) were negative. Also, drug resistant test was performed specifically for RIF to ascertain RIF resistant strain and the non-RIF resistant strains; only 6 (2.9\%) were resistant to RIF while 197 (96.6\%) were not resistant to RIF. Correlation analysis of MTB detected between sex, age and resistant to RIF showed nonsignificant association ( $p>0.05$ ). The prevalence rates include: $2.9 \%$ and $7.4 \%$ for RIF Resistance and Non-RIF resistance respectively. Chi square result reported about $90 \%$ non-significance and $10 \%$ significance which occurred in the counts between RIF resistant and MTB detected $(p<0.05)$.

Conclusion: The use of dependable molecular detection of MTB method has been able to provide strong epidemiological impetus for an enhanced MTB diagnosis and management to be encouraged and should be sustained, especially in resources limited regions like Nigeria, with massive challenges of prompt and accurate diagnosis of the infection. However, it is strongly believed that continuous leveraging on the potency of the molecular diagnostic approach will help to uncover the rifampicin resistance strains, which obviously would have been a huge challenge with the routine microscopic smear method.

Keywords: Mycobacterium tuberculosis; Rifampicin; Molecular detection; Limited resources region; Multi-drug resistance strain; Nigeria

\section{Obioma Azuonwu*, Ihua $\mathrm{N}$ and Kpomasiruchi W \\ Department of Medical Laboratory Science, Faculty of Sciences, Rivers State University of Science and Technology, Nkpolu, Port Harcourt, Nigeria}

Department of Medical Laboratory Science, Rivers State University of Science and Technology, Nkpolu, Port Harcourt, Nigeria.

Citation: Azuonwu O, Ihua N, Kpomasiruchi W (2017) Molecular Detection of Mycobacterium tuberculosis (MTB) and Rifampicin Resistant Strain among Subjects Accessing Health Care at Federal Medical Centre, Yenegoa, Bayelsa State; Nigeria. Transl Biomed. Vol. 8 No. 3:120 


\section{Introduction}

Tuberculosis (TB) caused by mycobacterium tuberculosis is the causative agent of a disease known as tuberculosis which has remained an utmost public health concern globally, according to 2013 World Health Organization (WHO) report. Nonetheless, it was reported that about one third of the world population is asymptomatically infected with latent mycobacterium tuberculosis [1]. Furthermore, Sub-Sahara region is severely affected massively and Nigeria with more than 150 million in population is one of the countries burdened with massive tuberculosis infection incidence. Nigeria is therefore, ranked number $13^{\text {th }}$ in the global scale of tuberculosis infected table [2].

Nonetheless, some strains of MTB are resistance to drugs and appear as mono resistant strain, multi or poly resistant strains. Multi drug-resistant (MDR) $M$. tuberculosis is described as an isolate resistant to at least two main first-line anti- TB drugs such as rifampicin (RIF) and Isoniazid (INH). Tuberculosis control and prevention programs are based on early diagnosis, followed by rapid identification of drug resistance strains [3]. A study by Lawson reported that multi drug resistant tuberculosis (MDR/TB) has implicated as much as $8 \%$ of all cultured specimens which were MDR/TB positive cases as recorded in three states in Nigeria [4]. Potentially, lots of awareness and campaigns have been on to reduce the incidence rate of the disease to the barest minimum, but TB elimination remains a critical massive challenge, due to some critical factors like the emergence and re-emergence of clinical forms of rifampicin and multi-drug- resistant strains [1] The increasing trend of Human Immunodeficiency Virus infection contribute massively towards worsening the health conditions of the subject's affected [1].

The first report of the global project on drug resistance surveillance outcome, showed data from 35 geographical settings from the periods of 1994-1996 [5] respectively. Drug resistance is a global issue and MDR-TB ranged from $0 \%$ to $14 \%$ in new cases and $0 \%$ to $45 \%$ in previously treated cases according to the reports $[6,7]$. This report affirmed the fact that drug resistant tuberculosis was a challenge given that MDR-TB ranged from $0 \%$ to $16 \%$ (median: $1 \%$ ) for new cases and from $0 \%$ to $48 \%$ (median: $9 \%)$ in treated cases. Furthermore, a later report of third data comprising of 77 geographical sites (1999-2002) represented $20 \%$ of the global total of new smear-positive TB cases [8]. Furthermore, Multi-Drug-Resistant TB (MDRTB) account for $84 \%$ of the 'retreatment" cases of TB) with this finding, early diagnosis appears to be the best strategy to control MDRTB [1]. However, culture-based drug susceptibility testing (DST) for TB takes as long as 90 days to complete, due to the slow growth of $M$. tuberculosis [9]. Diagnostic delay is an impediment to effective MDRTB management therefore; implementation, development of molecular approaches for rapid detection of antimicrobial resistance strains are better options to attenuate MDRTB burden as posited by Rieder and Trebucq. Also, study had shown gene mutations that confer drug resistance in MTB as a huge challenge to overcome among research scientist, and clinical stakeholders in general $[10,11]$.

The epidemiology of multidrug resistant tuberculosis has been reported in many countries surveyed, as reported by Farmer, the report revealed that MDR-TB mainly develops in the course of TB therapy [12] and it is most common as a result of inappropriate therapy, abuses like missing doses or failure to complete treatment regimen, wrong prescription, the wrong dose or length of time for taking the drugs is not always available and even when the drugs are of poor quality most times [12-14]. Nigeria is contending with about 2,700 cases of multidrug- resistant tuberculosis, yearly. There were estimated to be about 290,000 cases of MDR-TB in 2010. Furthermore, as of 2013, 3.7\% of new tuberculosis cases have multidrug resistant tuberculosis. High prevalence of this infection is much higher in those previously treated for tuberculosis. World Health organization estimates that there were about 0.48 million new MDR-TB cases in the world as at 2013 [15]. Failure to response or effectively recognize and treat patients with drug-resistant tuberculosis (TB), will lead to increased mortality rate, incidental out breaks and resistance to other anti-tuberculosis drugs in our communities [16]. Also, multi-drug-resistant (MDR) and extensively drug resistant (XDR) tuberculosis can be treated if identified accurately with early effective diagnostic approach [17].

Thus, an increasing global incidence of drug-resistant mycobacterium tuberculosis infection has promoted the critical need for more effective methods that can rapidly and effectively detect $M$. tuberculosis and identify drug-resistant strains, even in a reduced quantity in a clinical sample [18]. However, there is a dearth of research work on the potency of molecular detection of some antimicrobial resistant strains of mycobacterium tuberculosis in this region. Furthermore, research work with the application of molecular detection using gene xpert for detection of MTB and its resistant strain is not routinely done rather, mere smear microscopy method with high probability of low sensitivity and specificity, is always been applied and encouraged in our remote clinical laboratories. It is therefore, strongly believed that data generated from this study, would underpin the need for more robust and effective methods for the prognosis, diagnosis and management of the epidemic, especially the identification of RIF and multi-drug resistance strains in the midst of emerging and re-emergence of infectious diseases epidemic outbreak globally.

\section{Methods}

\section{Study design and setting}

This cross-sectional observational study was designed with 204 subjects in participation, randomly recruited for those that met the inclusion criteria, such as patients with case of some antimicrobial resistance strain of Mycobacterium tuberculosis. All study participants were attending clinic at Federal Medical Centre, Yenagoa. This study was undertaken in Federal Medical Centre between September 2016 to April 2017 in Yenegoa, Bayelsa state of Nigeria. This is a tertiary level health facility located in the heart of Yenagoa, Bayelsa State in the South-South part of Nigeria.

\section{Ethical consideration}

Ethical consideration was obtained from Rivers State University of Science and Technology and Federal Medical Centre ethical 
committee. Permission to conduct the study was sought from the Microbiology Department. In addition, a written informed consent was obtained from all subjects and confidentiality was highly upheld throughout the study period till date.

Inclusion and exclusion criteria: The inclusion criteria for this study includes those who are willing to participate by providing their samples freely and those who presented themselves as patients with long time duration of cough and chest pain and finally patients that are booked for AFB screening. Nonetheless, the exclusion criteria include those who are not willing to participate and patients that are not recommended for AFB assay screening.

\section{Sample size and sampling technique}

Sample size was determined using the single population prevalence formula according to Cochran. Proportionate Stratified random sampling technique was used for recruitment and a total of 204 subjects who literarily met the inclusion criteria were included in the study.

\section{Data collection}

A well-structured validated questionnaire was used in this study. Socio-demographics and medical history of the subjects were obtained using questionnaire. Both primary and secondary data were used. The detection of MTB though ranges from simple smear microscope to AFB, to culture and molecular assays. The World Health Organization endorses the use of genotypic methods that targets specific molecular mutations associated with resistance to individual drugs [19]. Basically, there are two commercially available techniques which were used namely: xpert MTB/RIF and Geno type MTBDR respectively. These to a large extent depend on lack of probe hybridization to wild-type loci, indirectly indicating the presence of mutations. The xpert MTB/RIF system is limited in detection of only RIF's resistance [19] and this present work was limited to xpert MTB/RIF system use. Sputum collection was collected by asking the patients to cough into an open mouth sample container to cough deep sputum cough to the container and bring to the laboratory. Blood was collected by venepuncture, placed into $5 \mathrm{ml}$ vacutainer tubes containing Ethylene Diamine Tetra Acetic Acid Kg (EDTA K K $_{3}$. MTB and antimicrobial resistance strain was performed using modules thermal and optical system (Cepheid), cartridge self-contained disposable, computer system software barcode scanner, optional accessories printer UPS according to the molecular technique of gene xpert system application approach. Nonetheless, high levels of precautionary measures were followed to reduce cross infection among the laboratory staff.

\section{Data analysis}

This involved the retrieval of the completely filled questionnaire; a reliability index of 0.89 (Cronbach) alpha was obtained. Data were collated into excel sheet and later analysed with SPSS version 21 to obtain frequency and percentage for descriptive statistics. Also, measures of association included chi square, correlation and risk estimates were performed in this study. A $p$-value $<0.05$ was considered to be statistically significant.

\section{Results}

This study included a total of two hundred and four (204) subjects accessing health care at FMC Yenegoa, Bayelsa state; Nigeria. The study participants had a mean age of $37.23 \pm 16.68$ years and were categorized based on sex, 93 (45.6\%) male and 111 (54.4\%) female subjects. The occupational status of the subjects showed that greater percentage of them were students 111 (54.4\%) followed by civil service workers 60 (29.4\%). In addition, this present study was centred on molecular detection technique which involved the use of Gene xpert to detect MTB. The study revealed that only 21 (10.3\%) were detected using the molecular technique while 183 (89.7\%) were not detected thus, were MTB negative with this method. Also, drug resistant test was performed specifically RIF to ascertain RIF resistant strain and the non-RIF resistant strains; only 6 (2.9\%) were resistant to RIF while 197 (96.6\%) were not resistant to RIF (Table 1). Correlation analysis of MTB detected using molecular technique and sex, age and resistant to RIF showed non-significant.

The prevalence rates as obtained in this present study were $2.9 \%$ and $7.4 \%$ for RIF resistance strain and non-RIF resistance strain respectively whereas, the prevalence of MTB in Yenogoa among the study participants ( $\mathrm{N}=204)$ comprising of both resistant and non-resistant strains was $10.3 \%$ (Tables $2 a-2 e)$.

The results of chi square distribution were used to measure association between variables; a cross tabulation of categorical data is presented in Table $\mathbf{2}$ showing the percentages of the various variables with "no/negative" responses greater compared to "yes/positive". Independent variables of sex, age, etc were tested in relation to MTB detection, RIF resistance etc. The result reported about $90 \%$ non-significance and $10 \%$ significance which occurred in the counts between RIF resistance and MTB detected $\left\{\chi^{2}=9.068, d f=2, P=0.011\right.$. $\left.(N=204)\right\}$.

Risk was estimated using odd ratio and relative risk (risk ratio) with variables of age, sex and RIF resistant independently as

Table 1 Frequency and Chi square distribution.

\begin{tabular}{|c|c|c|c|c|}
\hline $\begin{array}{l}\text { Variables/ } \\
\text { classification }\end{array}$ & Frequency (\%) & Correlation & P-value & Remark \\
\hline \multicolumn{2}{|c|}{ Sex } & \multicolumn{3}{|c|}{ MTB } \\
\hline Male & $93(45.6 \%)$ & 0.079 & 0.264 & $\mathrm{~N} / \mathrm{S}$ \\
\hline Female & $111(54.4 \%)$ & -- & -- & -- \\
\hline \multicolumn{5}{|c|}{ Age (Years) } \\
\hline 60 and Above & $21(10.3 \%)$ & -- & -- & -- \\
\hline 40-59 Years & $50(24.5 \%)$ & 0.01 & 0.887 & $\mathrm{~N} / \mathrm{S}$ \\
\hline 20-39 Years & $105(51.5 \%)$ & -- & -- & -- \\
\hline 1-19 Years & $28(13.7 \%)$ & -- & -- & -- \\
\hline \multicolumn{5}{|c|}{ MTB detected (Molecular technique) } \\
\hline Yes & $21(10.3 \%)$ & -- & \multicolumn{2}{|c|}{--} \\
\hline No & $183(89.7 \%)$ & -- & \multicolumn{2}{|c|}{--} \\
\hline \multicolumn{5}{|c|}{ RIF Resistant detected } \\
\hline Yes & $6(2.9 \%)$ & 0.037 & \multicolumn{2}{|c|}{$0.604 \mathrm{~N} / \mathrm{S}$} \\
\hline No & $197(96.6 \%)$ & -- & \multicolumn{2}{|c|}{--} \\
\hline Indeterminate & $1(0.5 \%)$ & -- & \multicolumn{2}{|c|}{--} \\
\hline Vote: RIF: Rifan & ; MTB: Myc & cterium & & \\
\hline
\end{tabular}


Table 2 Prevalence of MTB and prevalence of RIF resistance strain among studied population.

\begin{tabular}{|c|c|c|c|c|}
\hline N=204 & RIF resistance strain & Non-RIF resistance strain & Total MTB detected & No MTB detected \\
\hline Frequency & $6(28.6 \%)$ & $15(71.4 \%)$ & $21(100 \%)$ & $183(100 \%)$ \\
\hline Prevalence & $2.90 \%$ & $7.40 \%$ & $10.30 \%$ & $89.70 \%$ \\
\hline Note: RIF: Rifampicin; MTB: Mycobacterium & & & \\
\hline
\end{tabular}

Table 2a Results of Chi-square test and descriptive statistics for sex by MTB.

\begin{tabular}{|c|c|c|c|c|}
\hline \multirow{2}{*}{\multicolumn{2}{|c|}{ Classification }} & \multicolumn{2}{|c|}{ MTB } & \multirow{2}{*}{ Total } \\
\hline & & No & Yes & \\
\hline \multirow{2}{*}{ Sex } & Female & 102 (55.7\%) & 9 (42.9\%) & 111 (54.4\%) \\
\hline & Male & $81(44.3 \%)$ & 12 (57.1\%) & 93 (45.6\%) \\
\hline \multicolumn{2}{|c|}{ Total } & $183(100.0 \%)$ & 21 (100.0\%) & $204(100.0 \%)$ \\
\hline
\end{tabular}

Table $\mathbf{2} \mathbf{b}$ Results of Chi-square test and descriptive statistics for sex by RIF resistant.

\begin{tabular}{|c|c|c|c|c|}
\hline \multirow{2}{*}{\multicolumn{2}{|c|}{ Classification }} & \multicolumn{2}{|c|}{ RIF Resistant } & \multirow{2}{*}{ Total } \\
\hline & & No & Yes & \\
\hline \multirow{2}{*}{ Sex } & Female & 107 (54.0\%) & $4(66.7 \%)$ & 111 (54.4\%) \\
\hline & Male & 91 (46.0\%) & 2 (33.3\%) & 93 (45.6\%) \\
\hline \multicolumn{2}{|c|}{ Total } & 198 (100.0\%) & 6 (100.0\%) & 204 (100.0\%) \\
\hline
\end{tabular}

Table 2c Results of Chi-square test and descriptive statistics for age by MTB.

\begin{tabular}{|c|c|c|c|c|}
\hline \multirow{2}{*}{\multicolumn{2}{|c|}{ Classification }} & \multicolumn{2}{|c|}{ MTB } & \multirow{2}{*}{ Total } \\
\hline & & No & Yes & \\
\hline \multirow{4}{*}{ Age } & 1-19 Years & 25 (13.7\%) & $3(14.3 \%)$ & $28(13.7 \%)$ \\
\hline & 20-39 Years & 94 (51.4\%) & 11 (52.4\%) & 105 (51.5\%) \\
\hline & 40-59 Years & 47 (25.7\%) & $3(14.3 \%)$ & $50(24.5 \%)$ \\
\hline & 60 years and above & $17(9.3 \%)$ & $4(19.0 \%)$ & $21(10.3 \%)$ \\
\hline \multicolumn{2}{|c|}{ Total } & $183(100.0 \%)$ & $21(100.0 \%)$ & $204(100.0 \%)$ \\
\hline
\end{tabular}

Table 2d Results of Chi-square test and descriptive statistics for age by RIF resistance.

\begin{tabular}{|c|c|c|c|c|}
\hline \multirow{2}{*}{\multicolumn{2}{|c|}{ Classification }} & \multicolumn{2}{|c|}{ RIF Resistant } & \multirow{2}{*}{ Total } \\
\hline & & No & Yes & \\
\hline \multirow{4}{*}{ Age } & 1-19 Years & $27(13.6 \%)$ & $1(16.7 \%)$ & $28(13.7 \%)$ \\
\hline & 20-39 Years & $100(50.5 \%)$ & $5(83.3 \%)$ & 105 (51.5\%) \\
\hline & 40-59 Years & $50(25.3 \%)$ & $0(0.0 \%)$ & $50(24.5 \%)$ \\
\hline & 60 years and above & $21(10.6 \%)$ & $0(0.0 \%)$ & $21(10.3 \%)$ \\
\hline \multicolumn{2}{|c|}{ Total } & 198 (100.0\%) & $6(100.0 \%)$ & 204 (100.0\%) \\
\hline
\end{tabular}

Table 2e Results of Chi-square test and descriptive statistics for RIF resistant by MTB.

\begin{tabular}{|c|c|c|c|c|}
\hline \multirow{2}{*}{\multicolumn{2}{|c|}{ Classification }} & \multicolumn{2}{|c|}{ MTB } & \multirow{2}{*}{ Total } \\
\hline & & No & Yes & \\
\hline \multirow{3}{*}{ RIF Resistant } & No & 178 (97.3\%) & 19 (90.5\%) & 197 (96.6\%) \\
\hline & Yes & $5(2.7 \%)$ & $1(4.8 \%)$ & $6(2.9 \%)$ \\
\hline & Indeterminate & $0(0.0 \%)$ & $1(4.8 \%)$ & $1(0.5 \%)$ \\
\hline \multicolumn{2}{|c|}{ Total } & $183(100.0 \%)$ & $21(100.0 \%)$ & 204 (100.0\%) \\
\hline
\end{tabular}

against MTB detection at $95 \%$ confidence level. The odd of having the disease for sex in the ratio of female to male showed $\mathrm{OR}=1.679 \pm 0.674-4.180$; while the risk ratio obtained $\mathrm{RR}=1.055 \pm$
0.959-1.161 for the negative cohort whereas the positive cohort had $R R=0.628 \pm 0.277-1.426$ (Table 3). 
Table 3 Risk estimate.

\begin{tabular}{|c|c|c|c|}
\hline \multirow{2}{*}{ Variable/Classification } & \multirow{2}{*}{$\begin{array}{l}\text { Value } \\
\text { OR/RR }\end{array}$} & \multicolumn{2}{|c|}{ 95\% Confidence interval } \\
\hline & & Lower & Upper \\
\hline $\begin{array}{c}\text { Sex * MTB } \\
\text { Odds ratio for sex (female/male) }\end{array}$ & 1.679 & 0.674 & 4.180 \\
\hline $\mathrm{MTB}=\mathrm{No}$ & 1.055 & 0.959 & 1.161 \\
\hline MTB=Yes & 0.628 & 0.277 & 1.426 \\
\hline $\begin{array}{l}\text { Age* } \text { MTB } \\
\text { Odds ratio for age (1-40 years/greater than } 40 \text { years) }\end{array}$ & 1.000 & 0.384 & 2.606 \\
\hline $\mathrm{MTB}=\mathrm{No}$ & 1.000 & 0.906 & 1.104 \\
\hline MTB=Yes & 1.000 & 0.423 & 2.362 \\
\hline $\begin{array}{l}\text { RIF Resistant* MTB } \\
\text { Odds ratio for RIF resistant (no/yes) }\end{array}$ & 1.780 & 0.198 & 16.004 \\
\hline $\mathrm{MTB}=\mathrm{No}$ & 1.079 & 0.752 & 1.548 \\
\hline MTB=Yes & 0.606 & 0.097 & 3.804 \\
\hline
\end{tabular}

\section{Discussion}

The socio-demographic characteristics of age, gender, occupation, level of education and occupation were obtained in this study; the result showed more female than male however, there was no sex disparity following the report of the correlation relative to detection of MTB. The younger age group were more in this study compared to the older age group and so, the detection of MTB and HIV as recorded here were mainly from this young study participants moreover, the study reported strong correlation between the age of the subjects and the MTB; this is in agreement with the report of WHO (2009) which showed that TB has its highest burden among young adults [3]. However, the reason for this trend could probably be linked to social life style of the young stars that enjoys adventure and risk taking as a strong way of live. Less than three percent of the MTB detected appeared to be resistant to RIF furthermore, a correlation exist between MTB and RIF resistant, though there seems to be nonsignificant in practical terms.

Considering the prevalence rates obtained in this study, approximately twenty-one [20] subjects were positive for MTB as detected using the molecular technique which gave a general prevalence of MTB as $10.3 \%$ among the study population; also, prevalence rates as obtained in this present study were $2.9 \%$ and $7.4 \%$ for RIF resistance strain and non-RIF resistance strain MTB respectively. The $2.9 \%$ prevalence as reported in this present study is equivalent to the 2012 drug-resistant prevalence survey conducted in Nigeria, which showed a prevalence rate of $2.9 \%$ among new TB cases whereas, retreatment TB cases had $14 \%$ according to WHO (2012) First National TB Prevalence Survey [2]. This is comparable with WHO (2011) report of Nigeria having an estimated MDR-TB rate of $2.2 \%$ for new cases and $9.4 \%$ retreatment TB cases, which made Nigeria ranked $15^{\text {th }}$ among the 27 High Burden Countries for MDR-TB in the globe [20] Similarly, Idigbe reported $2 \%$ resistant to Rifampicin (RIF) in their study [20]. The prevalence of RIF non-resistant obtained in this study was in contrast with the report of Idigbe which is about $38 \%$ for
non-RIF resistant particularly resistant to Isoniazid (INH) [21].

The RIF resistance prevalence of $2.9 \%$ in $10.3 \%$ of MTB detected cases found in this study is contrary to Nwadioha study which had a prevalence of RIF resistance in the study as $13.9 \%$ (Retreated $11.3 \%$; Treatment naïve $2.6 \%$ ) in $21.5 \%$ of MTB detected cases, the treatment naive RIF resistance prevalence [22] however, is similar with this present study.

In addition, studies from different geographic zones have showed varied prevalence of RIF resistance which all differ from the prevalence obtained from this present study as reported by Akaninyene and Dinic $[23,24]$. Also, comparatively a study done in Nnewi, South East Nigeria shared a similar view (prevalence of RIF's resistance of $2.7 \%$ ) with this present study, although the general MDR-TB from their study was lesser $(7.7 \%)$ than that obtained here (10.3\%) Uzoewulu though, RIF resistance had the least prevalence when compared to isoniazid thus, it could be deduced that the non-resistant RIF strain might be isoniazid resistant strain and resistant to other TB drugs. The low RIF resistance as reported by this study, and previous study mentioned has a crucial implication for both national and global TB multidrug resistant diagnosis, because RIF incorporation in molecular technique is a vital indicator, for molecular detection of MDR-TB as seen in the gene xpert technology [20,25].

Multidrug resistant TB like the one reported in this study could be acquired as a result of wrong prescription, irregular supply of drugs, treatment noncompliance, lack of supervision and poor adherence to medical advice [26]. In addition, healthseeking behaviour is of great concern because of variation in health seeking behaviour arising from the stigma related with tuberculosis, particularly in the area of this present study. Social and cultural factors have also been implicated like the issue of alcoholism and other substance abuse, which are common among this study population. This was exemplified in a study which recorded that alcohol abuse/dependence was related with an eight-fold rise in drug resistance [27]. In the same way in another study, alcoholism was proven to be one of the 
predisposing factors for development of multidrug resistant TB [28]. On the other hand, Uzoewulu in their study posited that the only significant factor for the development of drug resistance and MDR was the history of previous anti TB treatment [25]. Other factors such as age and sex were not significantly related with drug resistant TB. Furthermore, it is important to know that poor adherence to TB drugs is another issue causing resistance and mutation (spontaneous genetic mutations). These afore mentioned risk factors inhibit the growth of organism vulnerable to a specific drug, that was initially in use at the same time allowing multiplication of drug-resistant organisms, which when transmitted to another person results to drug resistance [29]. The role of poverty as a strong setback cannot be overlooked as a key factor in health determinant outcome, thus promoting lack of access to effective health care delivery in our remote communities. Most times, lack of money for prompt access to laboratory diagnosis and purchase of drugs could be worrisome and self-limiting towards achieving quality health care services, especially among the inhabitants of the developing communities. Nevertheless, moribund functional health care system, lack of dedicated Health professional's due to poor enumeration and incentive has continued to affect the functioning capacity of the already existing weak Health care facilities. Furthermore, the irregular government health policies and regulations that appears weak in practical terms in implementation, is also massively implicated in worsening the already poor health care delivery system in Nigeria, even as the government and her relevant agencies lack evidence base visible commitments to the health of her citizens [30,31]. Things has to change very fast, if we must guarantee a healthy and effective work force that will engender development and protect our territory without fear of favour in time of adversity.

\section{Conclusion}

Gene xpert is a real-time PCR assay for Mycobacterium

\section{References}

1 World Health Organization (2013) the use of bedaquiline in the treatment of multidrug-resistant tuberculosis: Interim Policy Guidance. WHO/HTM/TB/. Geneva, Switzerland. p. 6.

2 WHO (2012) Report of First National TB Prevalence Survey, Geneva, Switzerland.

3 World Health Organization (2009) Policy statement. Non-commercial culture and drug susceptibility testing for rapid screening of patients at risk of MDR-TB. World Health Organization, Geneva, Switzerland.

4 Lawson L, Yassin MA, Thacher TD, Olatunji OO, Lawson JO, et al. (2007) Clinical presentation of adults with pulmonary TB with and without HIV infection in Nigeria. Scand J Infect Dis 10: 1-6.

5 World Health Organization (1997) Global tuberculosis programme. Global tuberculosis control. WHO Report 1997. WHO/TB. Geneva, Switzerland. p. 225.

6 WHO (2000) Multidrug and extensively drug-resistant TB (M/XDRTB): 2010 global report on surveillance and response, Geneva, Switzerland.

7 Espinal MA, Laszlo A, Simonsen L, Boulahbal F, Sang Jae Kim, et al. tuberculosis, that simultaneously detects rifampicin (RIF) resistance following its validation and endorsement by WHO (2010) for TB endemic and HIV endemic region. It is a useful and reliable diagnostic technique for MTB assay, hence should be encouraged to be a tool for prompt diagnosis especially in regions where muti-drug resistance strains poses a massive challenge to public health protection. The prevalence of MTB and RIF resistance as obtained in this present, have shown to be similar with the trend in Nigeria. Nonetheless, this study has been able to provide epidemiological basis for classification of this region as one of the study area that is leveraging on the skeletal support from some donor agencies that are in operation within the region. The need to scale up the much-needed technical support in this direction cannot be over emphasised, as the presence of the robust and more effective diagnostic tools are not readily available in our remote communities with large population of the vulnerable groups, who cannot potentially afford effective health care delivery services in the real sense of it.

Government needs to enhance TB (especially the drug resistant strain) diagnosis and management system in the country. Notably, other molecular diagnostic techniques which involve resistant to other drugs apart from RIF should be made available; as only few were resistant to RIF which was the only method used in this study, there could probably be other forms of resistance strains which could have been missed out, due to unavailability of other forms of diagnostic device.

\section{Acknowledgement}

We will always be grateful to Prof. SD Abbey, Dr Azuonwu, Goodluck, Brown Belema Joy and Mrs Enwereji Hope for their continuous support and prayers. We pray it will certainly be sustained.

(2001) Global trends in resistance to anti-tuberculosis drugs. N Engl J Med 344: 1294-1303.

8 WHO (2003) Treatment of tuberculosis: Guidelines for national programmes (3rd edn), Geneva, Switzerland.

9 Dalton T, Cegielski P, Akksilp S (2012) Prevalence of and risk factors for resistance to second-line drugs in people with multidrug-resistant tuberculosis in eight countries: A prospective cohort study. Lancet 380: 1406-1417.

10 Rieder HL, Cauthen GM, Comstock GW, Snider DE (1989) Epidemiology of tuberculosis in the United States. Epidemiol Rev 11: 79-98.

11 Cole ST (1997) Molecular basis of drug resistance in $M$. tuberculosis. The Wellcome Trust meeting on antibiotic resistance. UK.

12 Farmer $P$ (1999) Infections and inequalities: The modern plagues. Berkeley: University of California Press, USA.

13 Gao Qian, Li Xia (2010) Transmission of MDR tuberculosis. Drug Discovery Today: Disease Mechanisms 7: e61-e65.

14 Edlin BR, Tokars JI, Grieco MH (1992) An outbreak of multidrugresistant tuberculosis among hospitalized patients with the acquired immunodeficiency syndrome. N Engl J Med 326: 1514-1521. 
15 McCray E, Onorato IM (2000) The interaction of human immunodeficiency virus and multidrug resistant Mycobacterium tuberculosis. In: Bastian I, Portaels F (eds). Multidrug-resistant tuberculosis. Kluwer Academic Publishers, Netherlands. pp: 45-57.

16 Alberto M, Alberto R, Anna CC (2014) Extensively drug-resistant tuberculosis: Epidemiology and management. Clin Epidemiol 6: 111-118.

17 Mitnick CD, Shin SS, Seung KJ (2008) Comprehensive treatment of extensively drug-resistant tuberculosis. N Engl J Med 359: 563-574.

18 World Health Organization (2008) Anti tuberculosis drug resistance in the world fourth global report: The WHO/IUATLD global project on anti-tuberculosis drug resistance surveillance. Geneva, Switzerland.

19 World Health Organization (2010) Multi drug and extensively drug resistant TB (M/X DR-TB), Global report on surveillance and response, (WHO/HTM/TB/), Geneva, Switzerland. p. 3.

20 Cochran WG (1977) Sampling techniques (3rd edn) New York: John Wiley \& Sons, USA.

21 Idigbe EO, Duque JP, John EK, Annam O (1992) Resistance to antituberculosis drugs in treated patients in Lagos, Nigeria. J Trop Med Hyg 95: 186-191.

22 Nwadioha SI, Nwokedi EOP, Ezema GC, Eronini NC, Anikwe A, et al. (2014) Drug resistant Mycobacterium tuberculosis in Benue, Nigeria. Br Microbiol Res J 4: 988-995.

23 Akaninyene O, Victor U, Abdulrazak H, Soter A, Lovett L, et al. (2013) Drug resistance among pulmonary tuberculosis patients in Calabar, Nigeria. J Pulm Med 13: 6.

24 Dinic L, Akande P, Idigbe EO, Ani A, Onwujekwe D, et al. (2012)
Genetic determinants of drug-resistant tuberculosis among HIVinfected patients in Nigeria. J Clin Microbiol 50: 2905-2909.

25 Uzoewulu NG, Ibeh IN, Lawson L, Goyal M, Umenyonu N, et al. (2014) Drug resistant Mycobacterium tuberculosis in tertiary Hospital South East, Nigeria. J Med Microbiol Diag 3: 141.

26 Park WG, Bishai WR, Chaisson RE (2002) Performance of the microscopic observation drug susceptibility assay in drug susceptibility testing for Mycobacterium tuberculosis. J Clin Microbiol 40: 4750-4752.

27 Snider DE Jr, Long MW, Cross FS, Farer LS (1984) Six-months isoniazid-rifampin therapy for pulmonary tuberculosis. Am Rev Respir 129: 573-579.

28 Fleming MF, Krupitsky E, Tsoy M, Zvartau E, Brazhenko N, et al. (2006) Alcohol and drug use disorders, HIV status and drug resistance in a sample of Russian TB patients. Int J Tuberc Lung Dis 10: 565-570.

29 Khoharo HK, Shaikh IA (2011) Drug resistance patterns in pulmonary tuberculosis. J Pak Med Assoc 61: 229-232.

30 Azuonwu O, Nnenna I, Uwuma OE (2017) Evaluation of haematological profile of geriatric subjects in port Harcourt metropolis of Niger Delta of Nigeria. J Clin Lab Med 2: 111.

31 Azuonwu O, Nnenna I, Douglass AS, Ntaa NB (2016) Consequences of Haemolytic Disease of the Fetus and Newborn (HDFN) and the clinical significance of antibody screening in prenatal diagnosis: A Study of multigravidal and primigravidal women in Port Harcourt, Niger Delta. J Clin Lab Med 1: 106. 